\title{
Research on Cross-eye Jamming Technique based on Phase Diversity Processing
}

\author{
Yu Daobin ${ }^{1}$, Wang Hongyan ${ }^{2}$ and Wu Yanhong ${ }^{1}$ \\ ${ }^{1}$ The Equipment Academy, The Optoelectronic Equipment Department \&\& Science and Technology on Complex Electronic System \\ Simulation Laboratory, Beijing, China \\ ${ }^{2}$ The Equipment Academy, The Information Equipment Department \&\& Science and Technology on Complex Electronic System Simulation \\ Laboratory, Beijing, China
}

\begin{abstract}
The cross-eye jamming technique is a common method for monopulse tracking radar, which can cause a displacement towards the wrong direction and result in an angle deception. This paper proposes a modification of the traditional cross-eye jamming system, and the phase diversity processing of the received signal is introduced, and its precise expression of the offset is derived. The phase difference between the two branches of the signal processing is controllable, which is in the vicinity of $180^{\circ}$ phase shift, reducing the coordination requirements of the two branches. Simulation with different parameters is conducted so as to prove the conclusion, and the flexible control of the jamming effort is realized by this new cross-eye jamming technique.
\end{abstract}

\section{Introduction}

The monopulse tracking radar has strong ability against the traditional interference technology which poses a great challenge to the jamming part, and relevant research becomes urgent. An important way to interfere with the general monopulse tracking radar is the application of spatially separated sources of interference. The phase characteristic of spatially separated jammer may be coherent or irrelevant. Non-coherent sources of interference between phases mean a random relationship, and it's easy to be eliminated or tracked. Coherent interferences have determined or synchronous relationship between phases of multiple jammers and its apparent power may be located outside the centre of the constant angle, and it has less restrictions. Therefore, the application of coherent interference is an effective means for interference monopulse tracking radar [1-3].

Cross-eye interference is a typical coherent interference technology, domestic and foreign scholars have done some extensive researches. The model's analysis under cross-eye interference environment, the angle's control of aiming axis and system simulation for monopulse tracking radar are key issues, which are the cross-eye interference theoretical basis for studying and improving cross-eye interference structure [4-6]. Currently, the available methods for improving cross-eye such as by increasing the number of interference sources and divided branches are proposed. Although it can increase the performance of the system, but to a certain extent, also increase the complexity of interference control and system's construction $[7,8]$.
This paper presents a new method for cross-eye interference based on phase diversity processing, which can result in a flexible and controllable interference effects. On the basis of traditional cross-eye interference principle, the procedure of phase diversity processing and its structure are given. The obtained interference effect is derived from a mathematical model, and the conclusions are proved through related simulation finally.

\section{Analysis of Cross-eye Jamming}

Cross-eye interference takes advantage of the limited resolution of the angle tracking radar system, enabling optimum parameter's design for maximum directional error. So the stronger interference guides, the more that the radar pointing to the wrong target. Cross-eye interference is based on the front waveform's distortion effect. When the radar's tracking direction and the target's moving direction is in a certain relationship, a large tracking error will generate which causing a stronger displacement from the aiming point, creating plenty of false targets with different angles information. The false point is moving to a new location in the range of the target of interest, and it has nothing to do with the distance, but can achieve a large offset distance. Advantages of cross-eye jamming technology are ensured when the two sources of coherent radiation interference signal's phase are matched to $180^{\circ}$, but the angle that the radar signal reaches the jammer won't affects. In this case, the radar can not distinguish echoes and interfering signals, only to see a false target decade meters' far away from the real target and track it.

Traditional cross-eye jamming technology under all circumstances requires that the two interfering signals are 
transmitted to maintain the correct phase and power relationship. On one hand, the amplitude-frequency characteristics of the two channels are of high consistency requirements, and are particularly sensitive to the interference pattern of aircraft movement, allowing little yaw angle. A slight deviation will reduce interference effect greatly. On the other hand, when meeting the optimum conditions for interference, the two sources of interference power will offset mostly, resulting in the consequence that less interference will enter the radar receiver. Therefore, cross-eye interference technology based on phase diversity processing is proposed. Its principle is that the interference of one branch is given a plurality of diversity in the vicinity of $180^{\circ}$ phase shift, so the signal on the two paths will have phase differences, which can produce different offset distance in a certain range over a period of time. It is similar to the multipath effects, overcoming the disadvantages above and producing controlled angle deception jamming for monopulse tracking radar.

\section{Phase diversity processing and its jamming effects' analysis}

\subsection{New cross-eye interference system}

Cross-eye interference technology for monopulse tracking radar takes advantage of two independent forwarding antennas, and each antenna has a transmitting and receiving antenna connected by transmission lines to an amplifier generating the interference signal. Above all, the interference system has further improvement that the phase diversity processing module is brought in, which should be set on a branch circuit for phase diversity signal processing. By adding this module, the amplitude ratio and phase difference between two coherent sources of interference can be designed in any combination, resulting in different distances offset. By matching the magnitude and phase timely, interference can be achieved and produce more false angles information. Improved cross-eye interference system is as shown in Fig. 1:

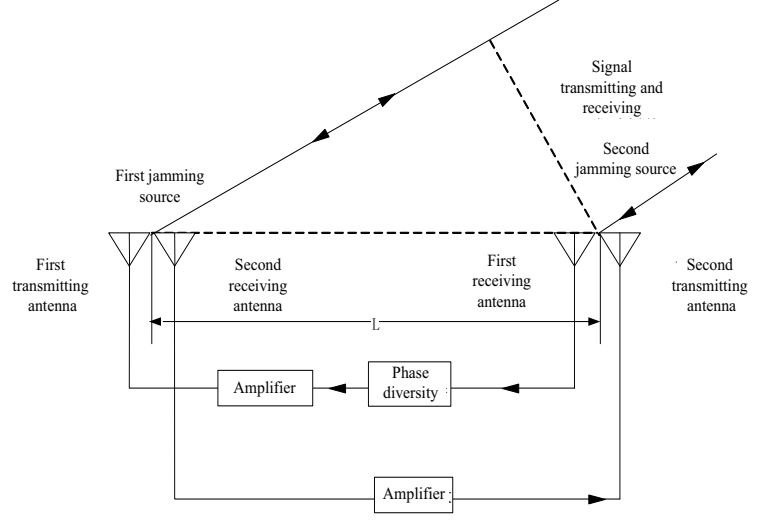

Figure 1. Schematic of Improved cross-eye interference system The cross-eye interference structure shown above functions as follows:

(1) The two independent interference sources obtain a monopulse tracking radar signals emitted by radar respectively;
(2) In the first branch, the obtain interference signal is amplified by power amplifier and transmitted back to the monopulse tracking radar;

(3)In the second radar, the obtained interference signal after phase diversity processing is also amplified by power amplifier and transmitted back as well;

After these interference signals with echo signals all received and processed by the monopulse tracking radar, a plenty of offset distance will generate. Near the target's real position, false targets can be made flexibly controlled, generating different angles decoy jamming effects.

\subsection{Phase diversity processing procedure}

By using the average logical method, the process can be depicted as follows:

(1) Signal splitter and phase modulation;

After receiving the radar signal, it's divided into $p$ branch $(p \geq 2)$ through a rectangular pulse train with equidistant spacing. Radar target echo signal on each branch is delayed to obtain a path of the sub-phase signal waveform from the same signal, and all sub-phase modulated signals from all the branches are set different time delayed so as to obtain phase modulated output in different period.

The original radar signal is expressed as:

$$
s(t)=\operatorname{rect}\left(t / T_{p}\right) \cdot e^{j 2 \pi\left(f_{0} t+\mu_{r} t^{2} / 2\right)}
$$

Different signal splitter have different delay characteristics, and the obtained signal of each branch is expressed as:

$$
\left\{\begin{array}{l}
s_{1}(t)=\operatorname{rect}\left(\frac{t-\tau_{1}}{T_{p}}\right) \cdot \exp \left\{j 2 \pi\left[f_{0}\left(t-\tau_{1}\right)+\mu_{r}\left(t-\tau_{1}\right)^{2} / 2\right]\right\} \\
s_{2}(t)=\operatorname{rect}\left(\frac{t-\tau_{2}}{T_{p}}\right) \cdot \exp \left\{j 2 \pi\left[f_{0}\left(t-\tau_{2}\right)+\mu_{r}\left(t-\tau_{2}\right)^{2} / 2\right]\right\} \\
: \\
s_{p}(t)=\operatorname{rect}\left(\frac{t-\tau_{p}}{T_{p}}\right) \cdot \exp \left\{j 2 \pi\left[f_{0}\left(t-\tau_{p}\right)+\mu_{r}\left(t-\tau_{p}\right)^{2} / 2\right]\right\}
\end{array}\right.
$$

After the phase modulation, the obtained signal can be written as follows:

$$
\left\{\begin{array}{l}
s_{1}^{\prime}(t)=s_{1}(t) \cdot e^{j 2 \pi p_{p}}=\operatorname{rect}\left(\frac{t_{r}-\tau_{1}}{T_{p}}\right) \cdot \exp \left\{j 2 \pi\left[f_{0}\left(t-\tau_{1}\right)+\mu_{r}\left(t_{r}-\tau_{1}\right)^{2} / 2+\phi_{1}\right]\right\} \\
s_{2}^{\prime}(t)=s_{2}(t) \cdot e^{j 2 \pi \tau_{2}}=\operatorname{rect}\left(\frac{t_{r}-\tau_{2}}{T_{p}}\right) \cdot \exp \left\{j 2 \pi\left[f_{0}\left(t-\tau_{2}\right)+\mu_{r}\left(t_{r}-\tau_{2}\right)^{2} / 2+\phi_{2}\right]\right\} \\
: \\
s_{p}^{\prime}(t)=s_{p}(t) \cdot e^{j 2 \pi \phi_{r}}=\operatorname{rect}\left(\frac{t_{r}-\tau_{p}}{T_{p}}\right) \cdot \exp \left\{j 2 \pi\left[f_{0}\left(t-\tau_{p}\right)+\mu_{r}\left(t_{r}-\tau_{p}\right)^{2} / 2+\phi_{p}\right]\right\}
\end{array}\right.
$$

(2) Signal samples

According to the sub-phase signal stored in the control logic, the signal sampling time is selected after the phase modulation process, which is selected based on pre-stored logic. The $n\left(2 \leq n \leq p, n, p \in \mathrm{Z}^{+}\right)$branch is sampled in real time to obtain phase-modulated sub-pulse signal. This process can be represented by the formula as follows: 


$$
\left\{\begin{array}{l}
s_{1}{ }^{\prime}(t)=s_{1}{ }^{\prime}(t) \bullet\left[\operatorname{rect}\left(\frac{t-t_{1}}{t_{1}}\right) * \sum_{i=0}^{N_{1}-1} \delta\left(t-\sigma_{i}\right)\right] \\
s_{2}{ }^{\prime}(t)=s_{2}{ }^{\prime}(t) \bullet\left[\operatorname{rect}\left(\frac{t-t_{2}}{t_{2}}\right) * \sum_{i=0}^{N_{2}-1} \delta\left(t-\sigma_{i}\right)\right] \\
: \\
s_{p}{ }^{\prime \prime}(t)=s_{p}{ }^{\prime}(t) \bullet\left[\operatorname{rect}\left(\frac{t-t_{p}}{t_{p}}\right) * \sum_{i=0}^{N_{p}-1} \delta\left(t-\sigma_{i}\right)\right]
\end{array}\right.
$$

At this point, the radar signal is split into $N$ sections, where $N_{1}+N_{2}+\cdots+N_{p}=N$.

(3) Signal synthesis

According to pre-stored control logic, $m\left(2 \leq m \leq n, m, n \in Z^{+}\right)$phase-modulated sub-pulse signals synthesize from $n$ selected sampled signal branches. The obtained interference signal after the sub-sampled radar pulses phase modulation in all signal path combine at last. The interference signal has the same pulse length compared with the original signal, and it's expressed as:

$$
J(t)=s_{1}{ }^{\prime \prime}(t)+s_{2}{ }^{\prime \prime}(t)+\cdots+s_{p}{ }^{\prime \prime}(t)
$$

Above is the phase diversity handling process. The process of phase diversity interference signals' generation is shown in Fig. 2.

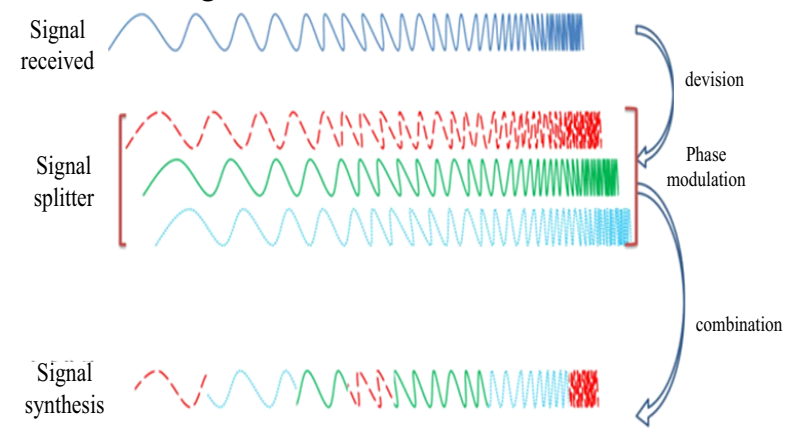

Figure 2. Schematic of Improved cross-eye interference system Schematic of phase diversity processing

\subsection{Interference Effect Analysis}

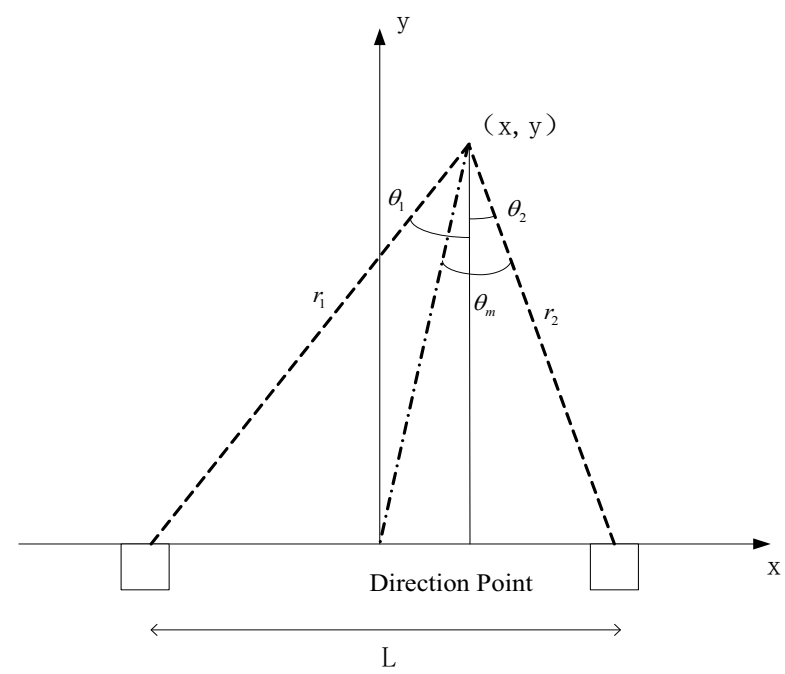

Figure 3. Interference of double coherent sources Mathematical model of double source interference is shown in Fig. 3. Provided the double sources of interference spacing $L$, the coherent interference source's measurement is given by the monopulse angle tracker:

$$
\Delta_{1}=k \theta_{1} \Sigma_{1}, \quad \Delta_{2}=k \theta_{2} \Sigma_{2}
$$

$k$ stands for the coordinate's factor. Thus the indicated $\theta_{i}$ of monopulse tracking angle equals to:

$$
\theta_{i}=\frac{1}{k} \frac{\Delta}{\Sigma}=\frac{\Delta_{1}+\Delta_{2}}{\Sigma_{1}+\Sigma_{2}}=\frac{\theta_{1} \Sigma_{1}+\theta_{2} \Sigma_{2}}{\Sigma_{1}+\Sigma_{2}}
$$

Set $\frac{\Sigma_{2}}{\Sigma_{1}}=a e^{j \Phi}$, the above formula can be further written as:

$$
\theta_{i}=\frac{\theta_{1}+\theta_{2} a e^{j \Phi}}{1+a e^{j \Phi}}
$$

Both sides multiply $\left(1+a e^{-j \Phi}\right) /\left(1+a e^{-j \Phi}\right)$, and apply Euler's formula to obtain the angle's value:

$$
\theta_{i}=\theta_{m}-\frac{\Delta \theta}{2} \frac{1-2 j a \sin \Phi-a^{2}}{1+2 a \cos \Phi+a^{2}}
$$

The real part of the equation represents the interference source's indication angle by double-coherent monopulse radar:

$$
\theta_{i}=\theta_{m}-\frac{\Delta \theta}{2} \frac{1-a^{2}}{1+2 a \cos \Phi+a^{2}}
$$

Imaginary part represents the direction of deviation angle, so offset distance is available at this time

$$
\Upsilon_{\text {miss }}=R \cdot \tan \left[\frac{\Delta \theta}{2} \frac{1-a^{2}}{1+2 a \cos \Phi+a^{2}}\right]
$$

With a small angle approximation $\tan (\cdot)$, considering $\Delta \theta=L \cos \Psi / R$ and thereby obtaining

$$
\Upsilon_{\text {miss }}=\frac{L \cdot \cos \Psi}{2} \frac{1-a^{2}}{1+2 a \cos \Phi+a^{2}}
$$

Where $a$ stands for the amplitude ratio of two coherent sources of interference, $\Phi$ stands for the phase difference between the sampled phase-modulated sub-pulse radar signal to the radar echo signal path during all jamming periods, and $\Psi$ stands for two interference source's connection angle between the vertical midpoint and radar sighting axis.

By phase diversity processing, different phases result in different offset distance's value at different times as follows:

$$
\frac{\Sigma_{2}}{\Sigma_{1}}= \begin{cases}a e^{j \Phi_{1}} & 0<t<t_{1} \\ a e^{j \Phi_{2}} & t_{1}<t<t_{2} \\ \vdots & \\ a e^{j \Phi_{n}} & t_{n-1}<t<T\end{cases}
$$

The final expression of offset distance obtained:

$$
\Upsilon_{\text {miss } i}(t)=\frac{L \cdot \cos \Psi}{2} \frac{1-a^{2}}{1+2 a \cos \left[\Phi_{i}(t)\right]+a^{2}}\left(t_{i-1}<t<t_{i}\right)
$$

The radar measures different offset distances in different time periods, and the interference effect obtained finally is shown in Fig.4: 


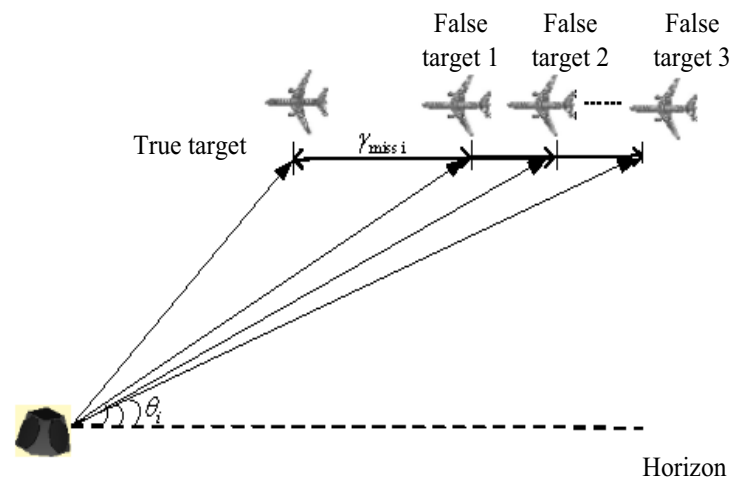

Figure 4. Monopulse tracking radar interference effect

\section{Simulation analysis}

Interference with airborne cross-eye system is simulated as an example to verify the above conclusion. Figure 5 shows the relationship between offset distance from the real target under different calculated amplitude ration and phase difference combination's variation, where $L=10 \mathrm{~m}, \theta_{i} \approx 4^{\circ}$.Assuming that the signal applied has achieved the desired signal interference ratio. Traditional cross-eye jamming technical parameters used are: $\Phi=180^{\circ}, \quad a=1$, then obtaining the maximum offset distance.

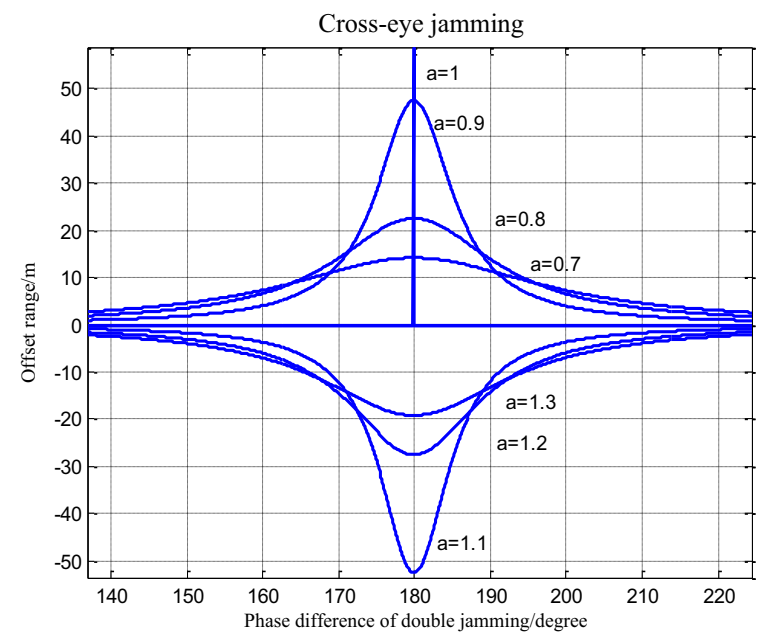

Figure 5. Change the offset range in cross-eye jamming

Through the phase diversity processing technique in a fixed amplitude ratio set, where different number of paths may be provided, five and eight branches applications are selected to be compared sharing average logic, respectively changing in the $\left[160^{\circ}, 200^{\circ}\right]$ and $\left[150^{\circ}, 200^{\circ}\right]$ spaced interval, and the obtained offset range's variation is shown in Figure 6. The phase offset is obtained from the variation in the diversity processing in $3 \mathrm{~s}$ by the random selection of each branch as shown in Figure 7.

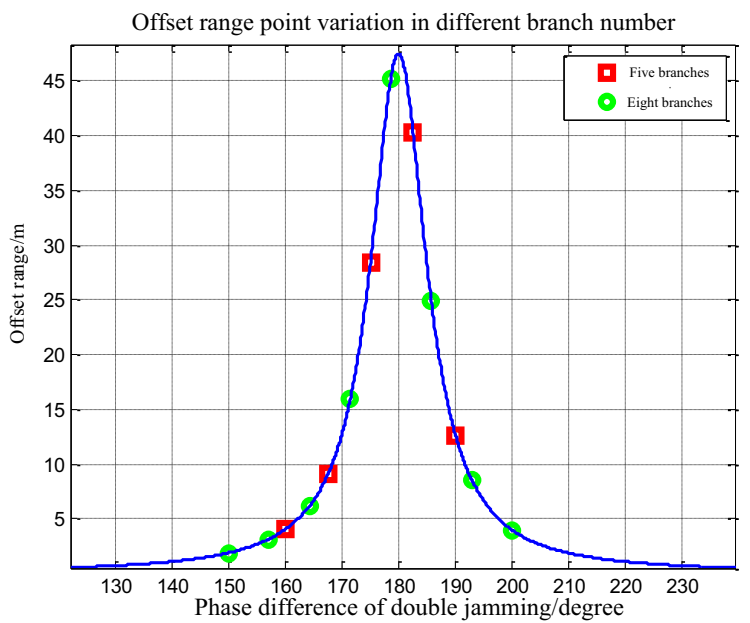

Figure 6. Offset range point variation of different branch number in fixed amplitude ratio

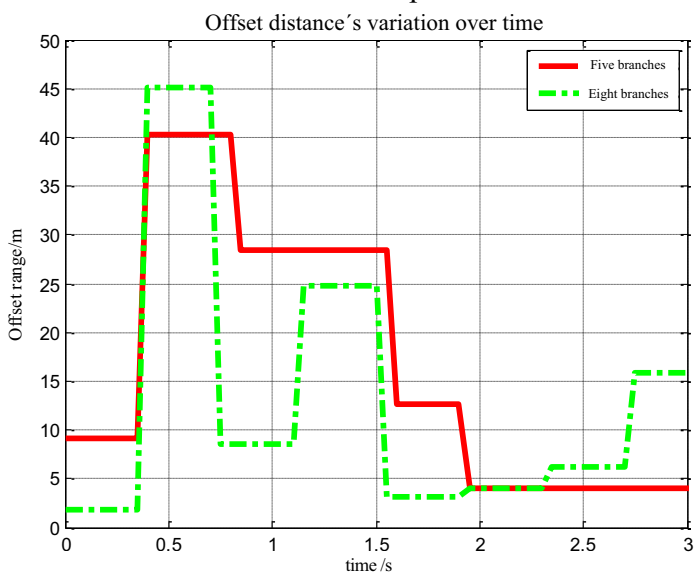

Figure 7. Offset distance's variation over time

In figure 6 , we can see that in a certain angle's region, the values are chosen in flexibility, causing different offset distance. The value's number is determined by the branches' number. The more the phase diversity processing branches are, the more values the offset range can be determined, no matter long or short, so as to reach a flexible jamming mode.

In figure 7, we can see that as time goes on, the offset ranging from $3 \mathrm{~m}$ to $45 \mathrm{~m}$. In the five branches case, it reaches $40 \mathrm{~m}$ during $0.3 \mathrm{~s}$ to $0.8 \mathrm{~s}$, and it's enough to form a false target compared with the range $L$ between the two interference sources. The eight branches case is more competitive than the former one, because it reaches a larger offset, and it shifts more frequently due to the advantages that more branches bring in.

By analysing the simulation results, some conclusions are listed as follows:

(1) The parameter set's selection process has great flexibility, where the number of branches and signal synthesis logic and so on can be flexibly combined to set, producing more false targets in short distance near the real target. In different periods it has different offset distances which are similar to the effect of multipath interference, generating multiple false targets flicker effect; 
(2) Since the phase diversity processing's parameter set can be treated with a certain regularity, so that the radar can not overcome the effects of angular error by just accumulating through the data, as a result that such interference is superior to the non-coherent multiple interference sources such as glint interference;

(3) The phase difference between the two branches of the signal processing is controllable, which is in the vicinity of $180^{\circ}$ phase shift to meet the requirements. It reduces the coordination requirements of the two branches, and will be better in practical engineering applications.

\section{Summary}

This paper presents a new cross-eye jamming technique based on phase diversity processing method through improvements in system architecture, and flexible and controllable interference effects can be achieved. This jamming method using phase diversity signal processing, generating a plenty of false targets with different false angles information, which has an obvious advantages compared with the traditional one. Less demanding on the system's coordination makes it easily realized. How to take advantage of the signal amplitude ratio of the two antennas to reduce the use of cross-eye antenna spacing requirements and get more improvement is one of the main directions of future research

\section{References}

1. Schleher D C. Electronic Warfare in the information age [M] America: Artech House, 1999: 256-271.

2. Falk L. Cross-eye jamming of monopulse $\operatorname{radar}[\mathrm{C}]$ Waveform Diversity and Design Conference. Pisa: IEEE Press, 2007: 209-213.

3. Meyer G J. Using cross-eye techniques to counter radio frequency agile monopulse [D]. Ohio: Air Force Institute of Technology, 1997: 156-168.

4. Cao Fei, Liu Qingyun, Xin Zengxian. Mathematical Modeling of Cross-eye Jamming[J]. Modern Radar, 2013, 35(6): 69-72.

5. Cheng Ning, Jiang Yong. Research on Angle Deception Using Interference of Coherent DualSources to Angle Tracking of Mono-Pulse[J]. Electronic Warfare, 2009, 126(3): 6-9.

6. Liu Yangtao, Hao Zhimei. Research on Jamming Simulation to Angle Tracking System of Monopulse Radar[J]. Aerospace Electronic Warfare, 2012, 28(4):62-64.

7. Harwood N.M., Dawber W.N., King D.J., et al Multiple-element Cross-eye[C]. IET Radar Sonar Navigation, 2007, 1(1): 67-73.

8. Wang Huiping, Zhang Youyi. A New Method to Realize Cross-eye Jamming Technique[J]. Warships Electronic Warfare, 2007, 30(6): 23-25. 